\title{
Asociación entre dependencia funcional y sintomatología afectivo-depresiva en pacientes en programa de hemodiálisis
}

María Mena Mayayo* - Eva Cerdán Urrutia* - Ana Pérez-Seoane Justo* - Joaquín Manrique Escola**

*Enfermera; ** Medico; Servicio de Nefrología. Unidad de Hemodiálisis. Hospital de Navarra. Pamplona

\section{Resumen}

La depresión es un problema muy prevalente en pacientes con enfermedad renal crónica en tratamiento de hemodiálisis. Por lo tanto, su valoración es importante no sólo desde el punto de vista diagnóstico, sino por su relación con una mayor morbimortalidad. El objetivo del presente estudio es valorar la relación entre dependencia física y sintomatología afectivo-depresiva, junto con otras variables demográficas. Para evaluar la presencia de sintomatología depresiva se utilizó la escala de Beck, para objetivar el grado de dependencia física se utilizó la escala de Barthel. Se aplicaron ambas escalas a una muestra de 94 pacientes en hemodiálisis hospitalaria.

Un 40\% mostró sintomatología depresiva, aunque alrededor del $25 \%$ se encontraba en tratamiento antidepresivo. Al valorar la escala de Barthel observamos que la dependencia media es moderada, aunque la mitad de los pacientes son independientes. Los pacientes en tratamiento antidepresivo obtuvieron un valor en la escala de Beck mayor que los que no los tomaban, por lo que serían susceptibles de ser valorados por un especialista para

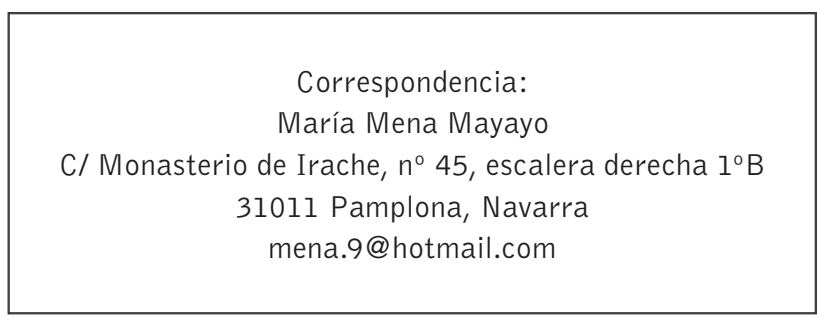

implantar algún tipo de terapia antidepresiva. La sintomatología depresiva es superior en el sexo femenino aumentando con la edad.

Pensamos que la escala de Beck podría contribuir a detectar aquellos pacientes susceptibles de valoración especializada encaminada a descartar patología depresiva o reevaluar la idoneidad de los tratamientos aplicados.

\section{PALABRAS CLAVE: \\ - DEPENDENCIA \\ - DEPRESIÓN \\ - HEMODIÁLISIS \\ - ESCALA DE BARTHEL \\ - ESCALA DE BECK}

\section{Association between functional dependency and affective-depressive symptomatology in patients on the haemodialysis programme}

\section{Abstract}

Depression is a very prevalent problem in patients with chronic renal disease undergoing haemodialysis. Accordingly, evaluating it is important not only from the diagnostic standpoint, but also because of its relationship with higher morbimortality. The aim of this study is to evaluate the relationship between physical dependency and affective-depressive symptomatology, together with other demographic variables. In order to evaluate the presence of depressive symptomatology, 
the Beck scale was used, to objectivize the degree of physical dependency, the Barthel scale was used. Both scales were applied to a sample of 94 patients undergoing dialysis at the hospital.

Depressive symptomatology was found in $40 \%$, although around $25 \%$ were receiving anti-depressive treatment. On evaluating the Barthel scale, we observed that the mean dependency is moderate, although half of the patients are independent. Patients receiving anti-depressive treatment obtained a higher score on the Beck scale than those who were not receiving such treatment, and therefore should be evaluated by a specialist to implement some kind of anti-depressive therapy. The depressive symptomatology is greater in females and increases with age.

We think that the Beck scale could contribute to detecting patients who should be referred to a specialist for evaluation aimed at ruling out depressive pathology or re-evaluating the suitability of the treatments applied.

\section{KEY WORDS:}

- DEPENDENCY

- DEPRESSION

- HAEMODIALYSIS

- BARTHEL SCALE

- BECK SCALE

\section{Introducción}

La depresión es un problema muy prevalente en pacientes con enfermedad renal crónica (ERC) en hemodiálisis (HD), aunque la cifra exacta no es conocida ${ }^{1}$ y se ha relacionado con una mayor morbimortalidad. Es importante distinguir entre presencia de sintomatología afectivo-depresiva y el diagnóstico de enfermedad psiquiátrica o depresión mayor. Para evaluar la presencia de sintomatología afectiva se han descrito diferentes escalas e índices, entre las que se incluye la escala de Beck ${ }^{2}$. Asimismo, la capacidad funcional de los pacientes en diálisis está limitada respecto a la población general y a otros grupos de población. Se han validado diferentes escalas para objetivar el grado de dependencia física, entre ellas la escala de
Barthel $^{3}$ que valora movilidad, capacidad para comer o higiene personal. Se ha descrito la estrecha relación entre estado de ánimo y capacidad física en diferentes grupos de estudio, incluso en pacientes con $\mathrm{ERC}^{4}$, sin embargo, son escasos los trabajos que hemos encontrado en pacientes en HD.

El objetivo del presente estudio basado en las escalas de Beck y Barthel, es valorar la relación entre dependencia física y sintomatología afectivo-depresiva, y otros factores que puedan estar relacionados con ellos en nuestra población de HD.

\section{Material y métodos}

Se trata de un estudio transversal realizado entre febrero y marzo del 2009. La población a estudio fueron 108 pacientes con ERC estadio 5 en programa crónico de HD. Los criterios de inclusión fueron: ser mayor de edad, estar incluido en HD al menos 2 meses antes, y haber dado consentimiento por escrito. Fueron excluidos los pacientes con deterioro cognitivo severo o enfermedad psiquiátrica previa diferente a depresión, los que estaban ingresados por cualquier motivo, analfabetos, u otras limitaciones físicas (sordera, ceguera), y la negativa del paciente a participar en el estudio. El consentimiento informado escrito y la hoja de información al paciente fueron realizados según los criterios del centro. Fueron excluidos por alteraciones cognitivas 7 pacientes y otros 7 pacientes rehusaron participar, quedando para el análisis 94 pacientes.

Para valorar el grado de dependencia en las actividades de la vida diaria (AVD) utilizamos la escala de Barthel ( $\mathrm{Ba}$ ), cuyos objetivos son: evaluar la capacidad funcional, detectar el grado de deterioro, monitorizar objetivamente la evolución clínica y diseñar planes de cuidados y de rehabilitación de forma interdisciplinar. Es un cuestionario heretoadministrado (personal enfermero) con un índice de fiabilidad alto (coeficiente alpha de Cronbach de 0,86-0,92) ${ }^{3}$, que evalúa diez actividades tipo "likert": comer, lavarse, aseo personal, vestirse, continencia urinaria y fecal, traslado a retrete, traslado cama-sillón, deambulación y subir-bajar escaleras. Los pacientes se clasificaron según su puntuación en: independientes (100 puntos), dependencia leve (91-99), moderada (61-90), severa (21-60) y total (0-20). 
Para valorar el grado de desesperanza (presencia de sintomatología depresiva), se utilizó la escala de Beck (Be) (fiabilidad elevada, coeficiente alfa de Cronbach de 0,83$)^{2}$. Es una escala autoadministrada de 20 preguntas con respuestas dicotómicas (si /no), con una puntuación entre 0 y 20 , definiéndose un grado de desesperanza elevado a un $\mathrm{Be} \geq 8$.

Se valoraron variables médicas y sociodemográficas. Entre las primeras: tiempo en HD, duración de la sesión, tipo de acceso vascular, presencia de diabetes mellitus, tratamiento antidepresivo, trasplante previo, e inclusión o no en lista de trasplante. Las variables socioeconómicas analizadas fueron: edad, sexo, estado civil (soltero, casado/pareja, viudo, separado/divorciado), situación laboral (activo/inactivo), lugar de residencia (urbano/rural), vivienda habitual (domicilio/institución) y el apoyo familiar.

Los resultados se presentaron como media \pm DE para variables paramétricas y mediana y RI para las variables no paramétricas. Se utilizaron los test de comparación de medias ( $t$ de Student y ANOVA) para las variables paramétricas, y análisis no paramétrico (Kruskal-Wallis y Wilcoxon) para el resto. Se utilizaron los coeficientes de Pearson y Spearman para la correlación (ajustado por factores de confusión). Se asumió significación estadística significativa para $p<0,05$ y muy significativo para $p<0,001$ Se utilizó el programa SPSS 14.0.

\section{Resultados}

La edad media del grupo fue de $67,9 \pm 14,1$ años (entre 23 y 87 años), con un tiempo medio de 29,6 \pm
26,5 meses en diálisis y una media de 4,1 $\pm 0,3$ horas por sesión ( 3 sesiones semanales) (tabla 1 ).

La media global de Beck fue de 7,09 $\pm 4,82$ y el $38,9 \%(n=41)$ de los pacientes presentaban síntomas depresivos ( $\mathrm{Be}>8$ ). La media de Barthel fue de 86,6 $\pm 26,6$ distribuido en: independencia $(n=49,52 \%)$; dependencia escasa $(n=17,18,1 \%)$; moderada $(n=15$, $16 \%)$; severa $(n=10,10,6 \%)$ y total $(n=3,3,2 \%)$. Destacamos de la distribución de Barthel que el 26,32\% de los pacientes tenían un grado de dependencia total para lavarse, el $18,8 \%$ necesitaban ayuda para vestirse, el $18,8 \%$ para trasladarse de la silla al sillón, el $28,2 \%$ para subir y bajar escaleras y el $25,3 \%$ para la deambulación (tabla 2).

\begin{tabular}{|c|c|c|}
\hline DESCRIPTIVOS & Media & Desv. Tipica \\
\hline \multicolumn{3}{|c|}{ n=108 Incluidos: 94. Excluidos: 7. Negativas: 7.} \\
\hline Edad (años) & 67,9 & 14,1 \\
\hline Tiempo en diálisis (meses) & 29,6 & 26,5 \\
\hline \multirow[t]{2}{*}{ Horas por sesión } & 4,18 & 0,38 \\
\hline & Si $(\%)$ & No $(\%)$ \\
\hline Mujeres & $25(26,6)$ & $69(73,4)$ \\
\hline FAVi & $43(45,7)$ & $51(54,3)$ \\
\hline DM & $21(22,3)$ & $73(77,7)$ \\
\hline Activo en lista espera & $19(20,2)$ & $75(79,8)$ \\
\hline Institucionalizado & $4(4,3)$ & 90 (9576) \\
\hline Urbano & $51(54,6)$ & $43(45,4)$ \\
\hline Apoyo familiar & $90(95,6)$ & $4(4,1)$ \\
\hline Con pareja estable & $63(67,0)$ & $31(33,0)$ \\
\hline Activo (laboral) & $3(3,1)$ & $91(96,9)$ \\
\hline Trasplante previo & $8(8,5)$ & $86(91,5)$ \\
\hline Antidepresivos & $18(19,1)$ & $76(80,9)$ \\
\hline
\end{tabular}

Tabla 1: Datos demográficos de la muestra

\begin{tabular}{|c|c|c|c|c|c|}
\hline \multirow{2}{*}{$\begin{array}{l}\text { Indice Barthel } \\
\qquad n=94\end{array}$} & \multicolumn{3}{|c|}{ Dependiente } & \multirow{2}{*}{$\begin{array}{l}\text { Total dependencia } \\
\qquad n=46(49 \%)\end{array}$} & \multirow{2}{*}{$\begin{array}{l}\text { Independiente } \\
\qquad \mathrm{n}=(51 \%)\end{array}$} \\
\hline & Total & \multicolumn{2}{|c|}{ Necesita Ayuda } & & \\
\hline Comer (n) \% & (1) $0,9 \%$ & \multicolumn{2}{|c|}{ (7) $6,5 \%$} & (8) $7,5 \%$ & (86) $92,4 \%$ \\
\hline Lavarse (n) \% & (28) $26,3 \%$ & \multicolumn{2}{|c|}{-} & (28) $26,3 \%$ & (66) $73,6 \%$ \\
\hline Vestirse $(n) \%$ & (6) $5,6 \%$ & \multicolumn{2}{|c|}{ (14) $13,1 \%$} & (20) $18,8 \%$ & (74) $78,8 \%$ \\
\hline Arreglarse $(n) \%$ & (8) $7,5 \%$ & \multicolumn{2}{|c|}{-} & (8) $7,5 \%$ & (86) $92,4 \%$ \\
\hline Ir al retrete $(n) \%$ & (4) $3,7 \%$ & \multicolumn{2}{|c|}{ (13) $12,2 \%$} & (17) $15,9 \%$ & (77) $84,6 \%$ \\
\hline Micción (n) \% & (5) $4,7 \%$ & \multicolumn{2}{|c|}{ (6) $5,6 \%$} & (11) 10,3 & (83) $89,6 \%$ \\
\hline Deposición (n) \% & (3) $2,8 \%$ & \multicolumn{2}{|c|}{ (8) $7,5 \%$} & (11) $10,3 \%$ & (83) $89,6 \%$ \\
\hline \multirow[t]{2}{*}{ Subir/bajar escaleras (n) \% } & (19) $17,8 \%$ & \multicolumn{2}{|c|}{ (11) $10,3 \%$} & (30) $28,2 \%$ & (64) $71,8 \%$ \\
\hline & & Gran ayuda & Mínima ayuda & & \\
\hline Traslado silla/sillón (n) \% & (1) $0,9 \%$ & (7) $6,5 \%$ & (12) $11,2 \%$ & (20) $18,8 \%$ & (74) $81,2 \%$ \\
\hline Deambulación (n) \% & (10) $9,4 \%$ & (14) 13,1 & (3) $2,8 \%$ & (27) $25,3 \%$ & (67) $74,7 \%$ \\
\hline
\end{tabular}

Tabla 2: Distribución de la dependencia para las AVD según la escala de Barthel 
Observamos una correlación entre ambas escalas muy significativa ( $r S=-0,50, p<0,001)$, manteniéndose esta relación al ajustar por toma de antidepresivos ( $r S=$ $-0,43, p<0,001)$. El valor de Beck fue significativamente superior en función del grado de dependencia y al dividir a los pacientes en dos grupos, aquellos con Barthel menor de 90 (dependencia importante o necesidad de ayuda para las AVD) tenían un Beck más alto $(10,24 \pm 3,85)$ que los pacientes con Barthel superior a 90 (dependencia escasa o no necesidad de ayuda) $(5,7 \pm 4,5, p<0,001$ ) (figura 1 ).

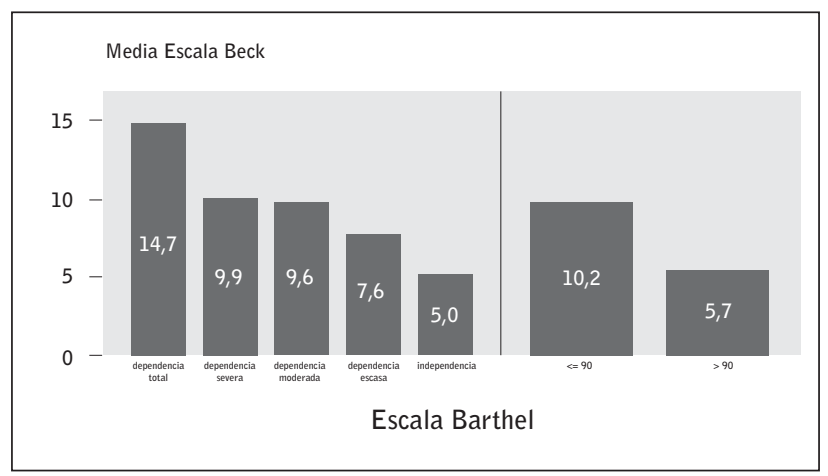

Figura 1: Valores de Beck según el grado de dependencia

Los pacientes en tratamiento antidepresivo presentaron puntuaciones de Beck y Barthel significativamente peores que los que no los tomaban $(p<0,05)$, de los 41 pacientes que tenían un Beck $\geq 8$ sólo el $26,6 \%$ tomaban antidepresivos.

Se valoraron las diferencias de Beck y Barthel en relación a otras variables, observando que las mujeres tenían un Beck significativamente superior (8,92 \pm $5,19, p<0,05)$ y los pacientes no incluidos en lista de espera $(7,75 \pm 4,53, p<0,005)$. Las mujeres mostraron mayor grado de dependencia según la escala de Barthel $(73,4 \pm 23,4, p<0,001)$, al igual que ocurría para los pacientes no incluidos en lista de espera $(84,0 \pm 23,5$, $p<0.001$ ). No encontramos diferencias para ambas escalas en el resto de variables valoradas (tabla 3 ). Observamos una correlación significativa de la edad con la escala de Beck $(r S=0,31, p<0,005)$ y con la escala de Barthel ( $r S=-0,30, p<0,005)$. No se observó asociación con el tiempo de diálisis ni con la duración de las sesiones en ninguna de las escalas.

\section{Discusión}

Las escalas de Beck y Barthel han sido ampliamente utilizadas en diversos grupos poblacionales, y nos han servido para definir la presencia de sintomatología afectivo-depresiva y capacidad funcional para las actividades de la vida diaria en nuestros pacientes. Una elevada proporción de pacientes en diálisis presenta algún tipo de dependencia, fundamentalmente física, y el número de pacientes que también presentan síntomas depresivos es creciente.

El estudio presentado nos ha mostrado que un $40 \%$ de los pacientes valorados presentan sintomatología depresiva, y de estos una escasa cuarta parte tomaba tratamiento antidepresivo. Al valorar la escala de Barthel, observamos que la dependencia media es moderada, y aunque la mitad de los pacientes son independientes, casi la otra mitad presentaba algún grado de dependencia, siendo hasta en un tercio de ellos de moderada a severa. La falta de autonomía en alguna de estas actividades tiene una repercusión directa sobre el funcionamiento de las unidades de diálisis

\begin{tabular}{|c|c|c|c|c|c|c|}
\hline & \multicolumn{2}{|c|}{ Indice de Beck } & \multirow[b]{2}{*}{$\boldsymbol{p}$} & \multicolumn{2}{|c|}{ Indice de Barthel } & \multirow[b]{2}{*}{$\boldsymbol{p}$} \\
\hline & Si & No & & Si & No & \\
\hline Mujer & $8,92 \pm 5,19$ & $6,39 \pm 4,47$ & $<0,05$ & $73,4 \pm 23,8$ & $91,8 \pm 19,0$ & $<0,001$ \\
\hline DM & $8,62 \pm 5,34$ & $6,65 \pm 4,57$ & ns & $80,7 \pm 27,3$ & $88,7 \pm 19,9$ & ns \\
\hline FAVi & $7,02 \pm 5,23$ & $7,13 \pm 4,44$ & ns & $92,9 \pm 17,2$ & $81,8 \pm 24,2$ & $<0,005$ \\
\hline Activo en lista & $4,42 \pm 4,99$ & $7,75 \pm 4,53$ & $<0,005$ & $98,4 \pm 5,78$ & $84,0 \pm 23,5$ & $<0,001$ \\
\hline Institucionalizado & $10,75 \pm 3,59$ & $6,92 \pm 4,78$ & $n s^{*}$ & $78,7 \pm 14,3$ & $87,2 \pm 22,1$ & $n s^{*}$ \\
\hline Apoyo familiar & $6,89 \pm 4,58$ & $11,5 \pm 7,85$ & $\mathrm{~ns}^{*}$ & $87,1 \pm 22,1$ & $82,5 \pm 17,5$ & $n s^{*}$ \\
\hline Con pareja estable & $6,62 \pm 4,77$ & $8,00 \pm 4,77$ & ns & $90,7 \pm 16,8$ & $79,1 \pm 28,4$ & ns \\
\hline Activo (laboral) & $4,67 \pm 2,51$ & $7,16 \pm 4,83$ & $\mathrm{~ns}^{*}$ & $100 \pm 0$ & $86,4 \pm 22,1$ & $n s^{*}$ \\
\hline Toma antidepresivos & $9,28 \pm 2,51$ & $6,54 \pm 4,66$ & $<0,05$ & $72,8 \pm 26,5$ & $90,2 \pm 19,4$ & $<0,05$ \\
\hline
\end{tabular}

* Escaso número de pacientes en el grupo Si.

Tabla 3: Valores de Beck y Barthel según variables estudiadas 
que atienden periódicamente a pacientes crónicos en régimen ambulatorio, constituyendo una población de pacientes que requieren cuidados específicos.

Ambas circunstancias, dependencia física y presencia de sintomatología depresiva se encontraban asociadas muy significativamente, tanto al correlacionar las variables como cuando subdividimos a los pacientes por grupos funcionales (pacientes que requieren ayuda y pacientes que no la requieren). Aquellos pacientes con mayor dependencia presentaron puntuaciones más altas en la escala de Beck. Este hallazgo puede guardar relación con lo descrito por otros autores en relación a la morbilidad añadida con el paso del tiempo en diálisis y con la aparición de síntomas físicos, que se asocian con trastornos emocionales y que a su vez influyen en el estado de salud percibido ${ }^{5}$. Sin embargo, revisada la literatura es escaso el número de trabajos que hemos encontrado donde se observe una asociación independiente entre ambas escalas como la descrita. A diferencia de lo descrito en otros trabajos ${ }^{4}$, no observamos peor estado de ánimo ni peor capacidad funcional en pacientes con más tiempo en diálisis, aunque sería razonable esperar este resultado a priori. Tampoco la duración de las sesiones influyó en los resultados.

Como cabía esperar, los pacientes de mayor edad presentaron mayor grado de dependencia y peores puntuaciones en la valoración del estado de ánimo. Cuando se utilizan medidas de calidad de vida relacionada con la salud basadas en puntuaciones según edad y sexo, se observa que el mantenimiento de la autonomía funcional es un factor clave que determina el bienestar de los pacientes ancianos en diálisis ${ }^{6}$ lo cual apoya la asociación observada entre dependencia física y estado de ánimo que mostramos en nuestro trabajo. La evaluación del grado de dependencia es por lo tanto importante, no sólo a la hora de valorar los recursos disponibles o aplicables, sino por ser una medida indirecta de calidad de vida y según lo expuesto, del estado de ánimo.

Los pacientes que estaban en tratamiento antidepresivo obtuvieron un valor en la escala de Beck mayor respecto a los que no lo tomaban, por lo que consideramos que los pacientes con puntuaciones elevadas ( $\mathrm{Be}>8$ ) serían susceptibles de ser valorados por un especialista que descartara la presencia de depresión o aplicara otro tipo de terapias ${ }^{7}$. El grado de dependencia o la sintomatología depresiva no variaron significativamente de unas áreas geográficas a otras ni se vieron influenciados por factores como tener pareja estable o apoyo familiar, a pesar de que se había descrito previamente que los pacientes con pareja presentan menos alteraciones psicosociales debido al apoyo que reciben ${ }^{8}$. El sexo femenino se asoció en nuestro trabajo a peores puntuaciones de Beck y de Barthel respecto de los hombres. Este resultado se observa también en otros estudios que evidencian que las mujeres tienen peor calidad de vida relacionada con la salud que los hombres ${ }^{4}$. Por último destacar el escaso número de pacientes activos laboralmente, institucionalizados o con transplante renal previo restó potencia estadística para el análisis en ambas escalas.

\section{Conclusión}

Hemos observado que la presencia de sintomatología depresiva valorada mediante la escala de Beck es elevada en pacientes en hemodiálisis, siendo superior en el sexo femenino y en pacientes de mayor edad. Un elevado porcentaje de pacientes en hemodiálisis es dependiente para las actividades de la vida diaria, siendo más acusada en edades avanzadas. Los pacientes con más discapacidad para las actividades de la vida diaria presentan mayor prevalencia de sintomatología depresiva, es decir, existe una asociación entre sintomatología depresiva y dependencia para las AVD. Pensamos que la escala de Beck podría contribuir a detectar aquellos pacientes susceptibles de valoración especializada encaminada a descartar patología depresiva o reevaluar la idoneidad de los tratamientos aplicados.

El elevado porcentaje de pacientes dependientes y con bajo estado de ánimo de las unidades de hemodiálisis supone una carga de trabajo extra para el personal sanitario y en concreto el de enfermería, haciendo en ocasiones imposible atender las necesidades de todos ellos. Esto, junto con la necesidad de atención integral de estos enfermos, hace que planteemos la utilización de escalas de uso rápido y sencillo, que permiten valorar estas circunstancias mediante parámetros objetivos, y descartar situaciones estrechamente relacionadas con aspectos psicosociales que influyen de manera determinante en la calidad de vida de nuestros pacientes. 


\section{Bibliografía}

1. Hedayati S, Bosworth H, Briley L, et al. Death or hospitalisation of patients on chronic hemodialysis is associated with a physician-based diagnosis of depression. Kidney International. 2008; 74: 930-636.

2. Sanz J, Vázquez C. Fiabilidad, validez y datos normativos del inventario para la depresión de Beck. Psicothema. 1998; 10: 303-318.

3. Cid-Ruzafa, Javier; Damián-Moreno, Javier. Valoración de la discapacidad física: el indice de Barthel. Rev. Esp. Salud Pública. 1997;.71 (2): 127-137.

4. Arenas MD, Moreno E, Reig A, Millán I y col. Evaluación de la calidad de vida relacionada con la salud mediante las láminas de Coop-Wanca en una población de hemodiálisis. Nefrología. 2004; 5: 470-479.
5. Álvarez Ude F, Fernández-Reyes $M$, Vázquez A y col. Síntomas físicos y trastornos emocionales en pacientes en programa de hemodiálisis periódicas. Nefrología . 2004; 36(1): 111-113.

6. Antoine V, Edy T, Souid M et al. Aging, the beginning of dialysis, the beginning of dependence: repercussions on the psychopathology of the very old dialysis patient. Nephrologie. 2004; 25: 83.

7. Cohen $S$, Norris L, Acquaviva K, Peterson R, Kimmel. Screening, Diagnosis, and Treatment of Depression in Patients with End-Stage Renal Disease.. Clin J Am Nephrol 2007; 2: 1332-1342.

8. Akman B, Ozdemir FN, Sezer S, Micozkadioglu H, Haberal M: Depression levels before and after renal transplantation. Transplant Proc. 2004; 36 (1): 111-113. 